\title{
Minimizing Hemodialysis Catheter Dysfunction: An Ounce of Prevention
}

\author{
Timmy Lee, ${ }^{1}$ Charmaine Lok, ${ }^{2}$ Miguel Vazquez, ${ }^{3}$ Louise Moist, ${ }^{4,5}$ \\ Ivan Maya, ${ }^{6}$ and Michele Mokrzycki ${ }^{7}$ \\ ${ }^{1}$ University of Cincinnati, Cincinnati, OH 45267-0585, USA \\ ${ }^{2}$ Faculty of Medicine, University of Toronto, University Health Network-Toronto General Hospital, Toronto, ON, Canada M5G 2C4 \\ ${ }^{3}$ University of Texas Southwestern Medical Center, Dallas, TX 75390-8856, USA \\ ${ }^{4}$ Department of Epidemiology and Biostatistics, University of Western Ontario, London, ON, Canada N6A 5W9 \\ ${ }^{5}$ Division of Nephrology, Department of Medicine, University of Western Ontario, London, ON, Canada N6A 5W9 \\ ${ }^{6}$ Nephrology Associates of Central Florida, Lake Mary, FL 32746, USA \\ ${ }^{7}$ Albert Einstein College of Medicine, Bronx, NY 10467, USA
}

Correspondence should be addressed to Michele Mokrzycki, mokrzm@aol.com

Received 29 September 2011; Accepted 10 October 2011

Academic Editor: Alexander Yevzlin

Copyright (C) 2012 Timmy Lee et al. This is an open access article distributed under the Creative Commons Attribution License, which permits unrestricted use, distribution, and reproduction in any medium, provided the original work is properly cited.

The maintenance of tunneled catheter (TC) patency is critical for the provision of adequate hemodialysis in patients who are TC-dependent. TC dysfunction results in the need for costly and inconvenient interventions, and reduced quality of life. Since the introduction of TCs in the late 1980s, heparin catheter lock has been the standard prophylactic regimen for the prevention of TC dysfunction. More recently, alternative catheter locking agents have emerged, and in some cases have shown to be superior to heparin lock with respect to improving TC patency and reducing TC-associated infections. These include citrate, tissue plasminogen activator, and a novel agent containing sodium citrate, methylene blue, methylparaben, and propylparaben. In addition, prophylaxis using oral anticoagulants/antiplatelet agents, including warfarin, aspirin, ticlodipine, as well as the use of modified heparin-coated catheters have also been studied for the prevention of TC dysfunction with variable results. The use of oral anticoagulants and/or antiplatelet agents as primary or secondary prevention of TC dysfunction must be weighed against their potential adverse effects, and should be individualized for each patient.

\section{Introduction}

Tunneled catheters (TCs) are frequently used in patients who require both temporary and long-term hemodialysis (HD) but do not have a functioning arteriovenous fistula, graft, or peritoneal dialysis catheter [1]. About $20 \%$ of prevalent and $80 \%$ of incident HD patients in the United States use a TC and the proportion is even higher in some other countries $[2,3]$. TCs have advantages and disadvantages as a vascular access for dialysis $[1,3,4]$. There is no need for a surgical procedure to place a TC or waiting for maturation prior to use. Thus, TCs are immediately available for use, and there are several different options on where to place them in most patients. Unfortunately, TCs have several major problems including frequent TC dysfunction and infections. Patients with TC have more hospitalizations, incur higher costs, and are at increased risk for inadequate dialysis and higher morbidity and mortality [1-6]. The main objective of this paper is to evaluate potential interventions to prevent $\mathrm{HD}$ TC dysfunction. According to the NKF Dialysis Outcomes Quality Initiative (KDOQI)-2006, dialysis access dysfunction is defined as an inability to achieve a dialysis blood flow rate of at least $300 \mathrm{~mL} / \mathrm{min}$ during the first hour of dialysis despite at least one attempt to increase blood flow [1]. Several interventions including instillation of locking solutions and administration of systemic anticoagulation and antiplatelet agents are reviewed. Table 1 is a summary of the most rigorously published clinical trials of interventions to prevent dialysis TC dysfunction [7-27]. 


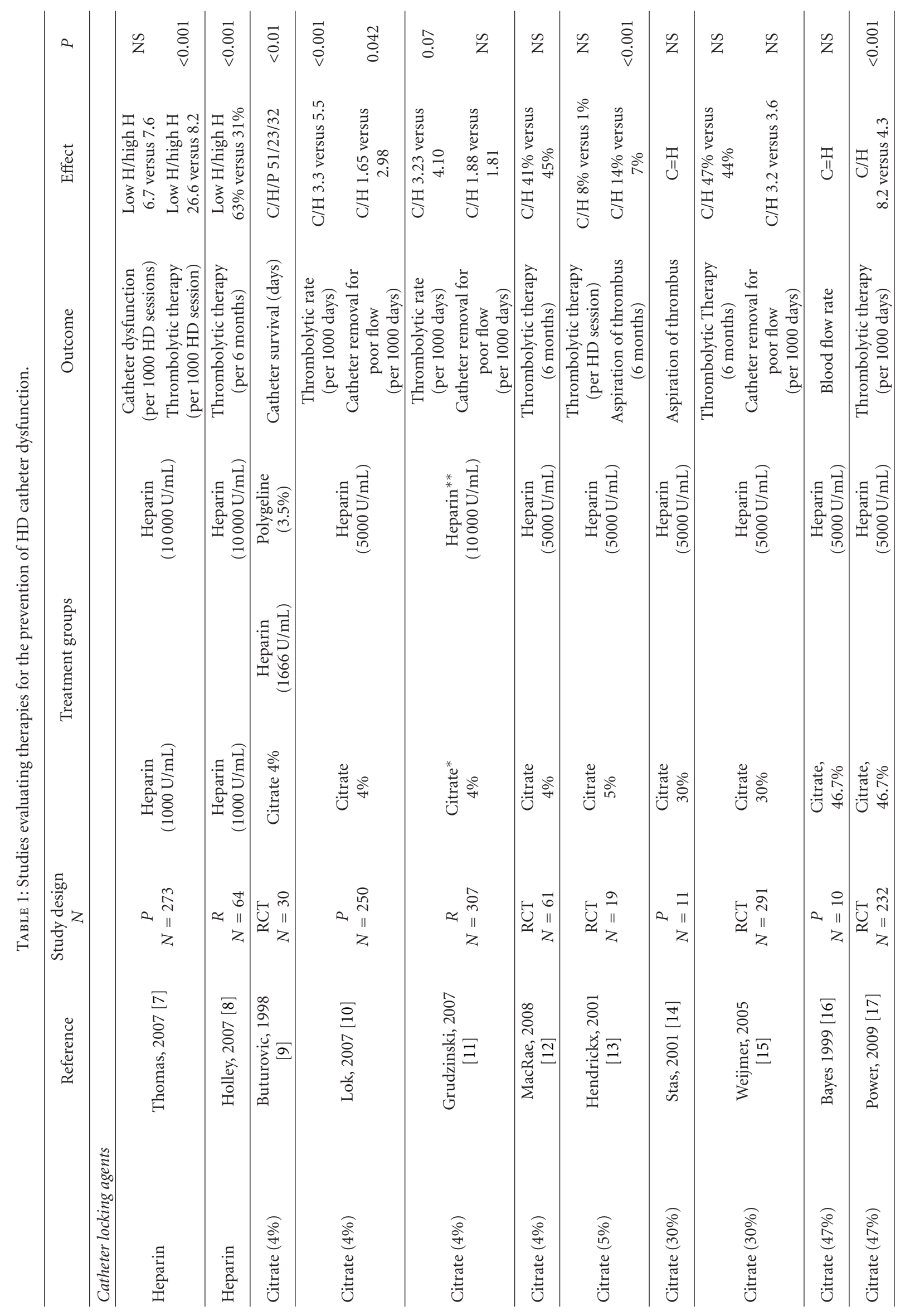




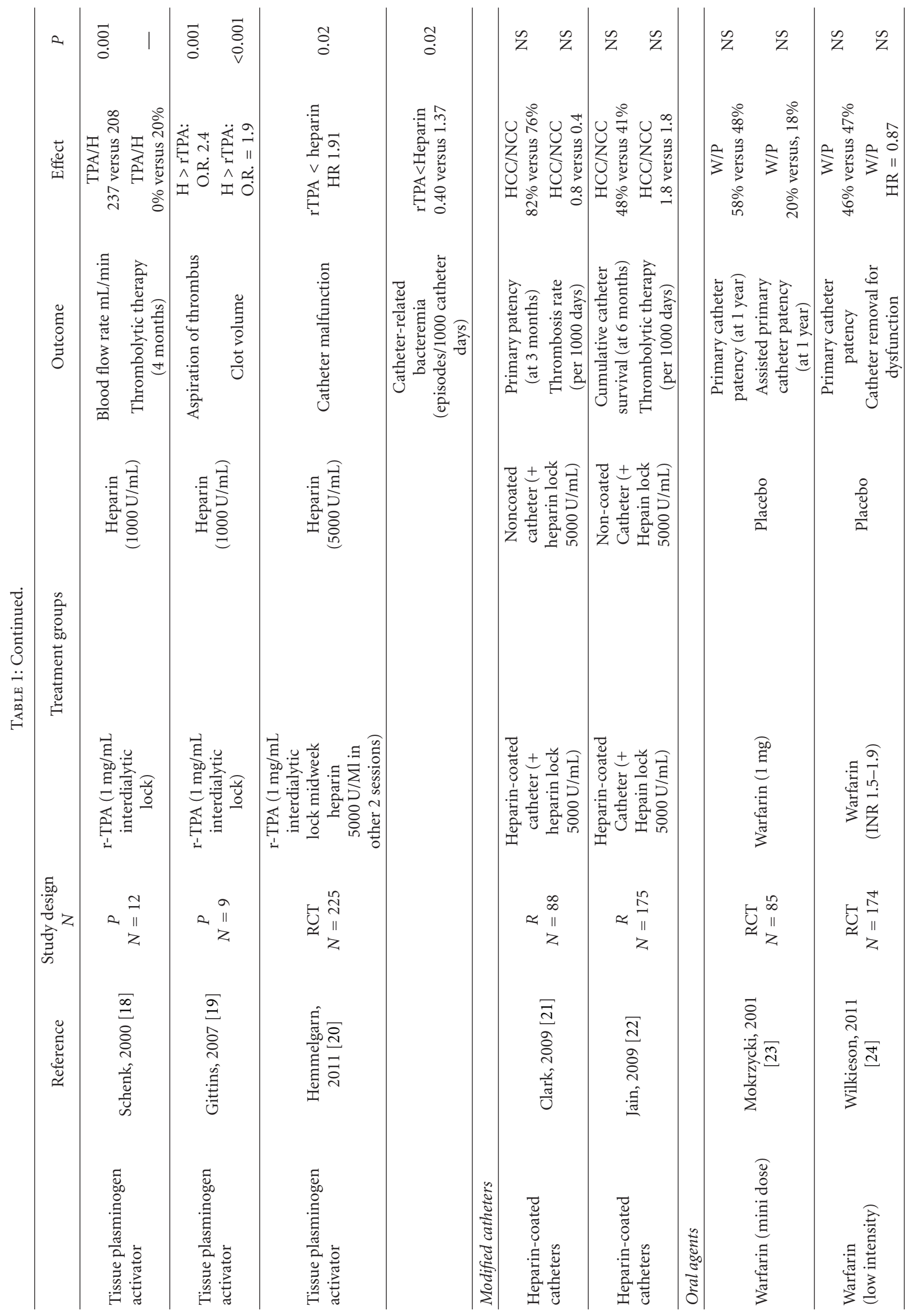




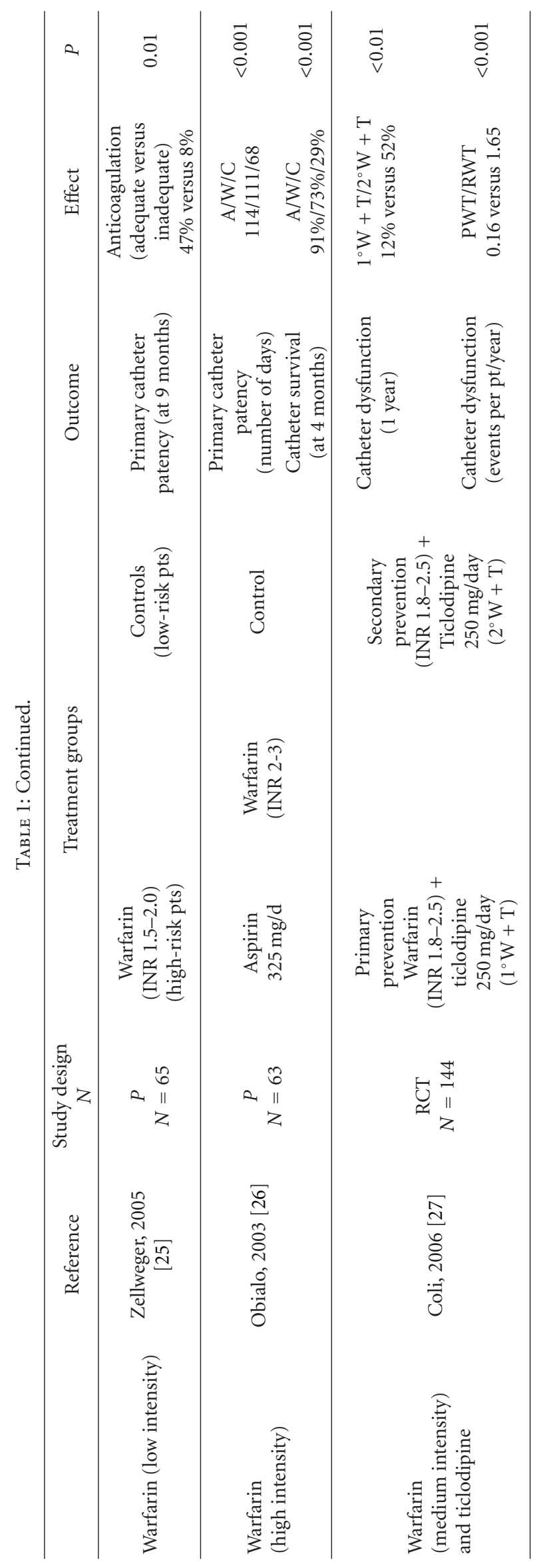




\section{TC Insertion}

Several investigators have reviewed the principles of TC insertion $[6,28-30]$. TC insertion should be performed with ultrasound guidance to identify the vein and guide the needle puncture $[6,31,32]$. The right internal jugular vein is the preferred site as it offers a more direct route to the right atrium and usually allows a smooth wide curvature to the TC $[1,6,28,29]$. The use of fluoroscopy is strongly recommended to guide placement of the TC tip in the midatrium $[1,28]$. TCs in the left internal jugular vein have a poorer blood flow than in the right internal jugular vein. TC in the subclavian vein can lead to subclavian vein stenosis and prevent future use of the ipsilateral upper extremity for a permanent vascular access [33]. Other sites such as femoral veins are not recommended as sites for TC insertion given the risks of infection and limitations to patient mobility.

\section{The Role of Biofilm and Fibrin Sheath Formation in Hemodialysis Catheter Dysfunction}

A biofilm is defined as a microbially derived, sessile community, characterized by cells that are attached to a substratum or to each other and are protected by a matrix of extracellular polymeric substances that they have produced [34]. One major complication of biofilms, in addition to infection, is the development of a fibrin sheath, which plays a major role in TC dysfunction.

While the pathophysiology of biofilm production is unclear, it has been hypothesized that biofilm formation occurs after initial contact of free-floating bacteria with a foreign surface, that is, dialysis TC, and can occur as early as 1-14 days after placement [35-40]. The bacteria initially attache and adhere irreversibly to the foreign surface, generate molecular signaling, and proliferate to transform into bacterial microcolonies [35-37]. Subsequently, the bacteria generate a coating of exopolysaccharide from the bacterial products so that their progeny can adhere firmly to the surface covered by a sticky glycocalyx matrix, called the biofilm, which envelopes the community of bacterial microcolonies [35-37]. Biofilms subsequently evolve and mature into a community of bacteria covered by a dense layer of matrix [35-37]. Furthermore, bacteria form biofilms preferentially in very-high-shear environments, similar to conditions that occur within the TC during the dialysis procedure, by enhancing bacterial adhesion [34]. Of note, the mere presence of a biofilm does not necessarily lead to infection and it may grow too slowly to produce clinical symptoms such as fever, chills and bacteremia. However, one important noninfectious complication of biofilm formation is the concurrent development of a fibrin sheath, which plays a major role in TC dysfunction.

Since the pathogenesis of biofilm development to fibrin sheath formation is not well understood, there is debate as to the sequence of fibrin sheath formation, biofilm development, and their interdependency [41]. However, the best evidence supports the hypothesis that a biofilm evolves over days to months into a more complex structure, a fibrin sheath. In addition to fibrin, these sheaths contain multiple other molecular and cellular components, including laminin, fibronectin, collagen, and smooth muscle cells, overlying endothelial cells [35]. These findings demonstrated that the TC-related fibrin sheath is an active response of the components of the vessel wall to the TC (e.g., biofilm formation) and associated thrombosis, as opposed to a mere deposition of acellular material and thrombus [42]. The fibrin sheath initiates at the point of contact between the TC and the vessel wall, advances along the entire length of the TC or device, and can create a one-way valve mechanism, with resultant decrease in the TC flow [42]. Fibrin sheaths play a major role in TC malfunction [35, 43-45]. Although the reported incidence of fibrin sheath can be as high as $100 \%$, this condition may remain subclinical. However, in clinically affected (symptomatic) patients, the fibrin sheath may result in thrombus formation and malfunctioning TCs, in addition to infection $[42,43]$.

Once biofilms form, they are very resistant to antithrombotic and antimicrobial agents. There have been several recent clinical studies that have focused on investigating prophylactic pharmacologic treatment with antithrombotic and antimicrobial locking solutions and biological characteristics of bacterial TC adherence and biofilm formation showing some promising results $[46,47]$. As with the paucity of therapies to prevent biofilm development, there are also few proven therapies to prevent fibrin sheath formation. The current treatment paradigms for management of fibrin sheaths in dysfunctional TCs include thrombolytic therapy, angioplasty with fibrin sheath disruption, and TC exchange $[1,42]$. Transfemoral percutaneous fibrin sheath stripping is associated with poor patency. The best management strategy for TC malfunction is its prevention. The most common form of prevention is with the use of anticoagulant locking during the intradialytic period.

\section{Heparin Catheter Lock}

Heparin lock has been used for decades with relative safety; however, TC dysfunction continues to remain problematic. The rate of TC thrombosis associated with heparin lock in several large series ranges between 4-5.5 episodes/1000 days, and the rate of TC loss due to dysfunction is 1.8$3.6 / 1000$ days [7-12]. The concentration of heparin used as a TC locking solution varies among studies, ranging between $1,000 \mathrm{U} / \mathrm{mL}$ and $10,000 \mathrm{U} / \mathrm{mL}$. In a prospective trial comparing a period of high dose $(10,000 \mathrm{U} / \mathrm{mL})$ versus a period of low dose $(1,000 \mathrm{U} / \mathrm{mL})$ heparin lock, there was no significant difference in TC dysfunction or bleeding complications between heparin lock concentrations, although the need for TPA therapy was 4 -fold higher $(P<0.001)$ when low-dose heparin lock was utilized [7]. Similar results were reported in a smaller, retrospective study comparing TPA administration in $2 \mathrm{HD}$ units, one unit using highdose heparin lock $(10,000 \mathrm{U} / \mathrm{mL})$ and the other using lowdose heparin lock $(1,000 \mathrm{U} / \mathrm{mL})$ [8]. Low-dose heparin lock was associated with 2 -fold increase in TPA administration 
$(P<0.001)$. Theoretically, the use of high-concentration heparin lock may be advantageous in reducing both TPArelated costs and delay in treatment initiation; however, when Holley and Bailey took into account the differential cost/treatment between low- and high-dose heparin lock (\$0.20 versus \$2.67), low dose heparin resulted in significant savings despite higher TPA use [8]. Low-dose heparin lock $(1000 \mathrm{U} / \mathrm{mL})$ is particularly advantageous in the immediate postinsertion period [21]. In a retrospective analysis comparing heparin lock $(5000 \mathrm{U} / \mathrm{mL})$ versus heparin lock $(1000 \mathrm{U} / \mathrm{mL})$ or citrate administered immediately after TC insertion, patients receiving high-dose heparin lock $(5000 \mathrm{U} / \mathrm{mL})$ had a 9-fold increase in composite bleeding events $(P=0.01)$, and $7.7 \%$ experienced a major bleeding event (versus $0 \%$ in the low-dose heparin or citrate group) [48]. A significant increase in severe hemorrhage after TC insertion was associated with heparin lock $(5,000 \mathrm{U} / \mathrm{mL})$ in a large randomized controlled trial in comparison to citrate $30 \%$ lock, (rate of severe hemorrhagic events: heparin 13\% versus citrate $4 \%, P=0.005)$ [15].

The optimal volume of heparin lock should be individualized according to the patient and catheter characteristics. All dialysis TCs have some degree of locking solution leakage, depending on their design, even when $<20 \%$ of the TC lock volume is instilled [49]. TC leak begins immediately after instillation and continues over a 30 -minute period, and is higher in nontunneled catheters for both periods $[50,51]$. The excess leakage volume is $0.16-0.48 \mathrm{~mL}$ with $<20 \%$ TC fill volume, and $0.99-1.43 \mathrm{~mL}$ with $>20 \%$ TC fill volume. TC lock overfill by $20 \%$ has been suggested to ensure delivery of heparin to the TC tip and wall and to improve TC patency which may be desirable in TCs with a history of recurrent thrombosis. Overfill by $20 \%$, however, may result in the inadvertent systemic administration of substantial amounts of heparin and may be problematic in the preoperative patient, or in patients with bleeding diatheses [49, 52]. Undesired systemic anticoagulation (increased activated partial thromboplastin time), caused by heparin lock overfill may persist for up to 4 hours [53]. The average rate of major bleeding episodes associated with heparin lock $(5,000 \mathrm{U} / \mathrm{mL})$ is approximately $2 / 1000$ TC days [15].

Heparin lock has been traditionally administered in a thrice weekly dosing, with an interdialytic heparin dwell period. The results of a recently published small prospective study reported improved efficacy using a 6-day per week heparin locking regimen. This protocol is not practical in an outpatient HD setting, but may be useful in hospitalized patients with HD TCs. One caveat to this protocol, however, is that more frequent access of the TC lumen has the potential to increase the risk of infectious contamination if not using sterile procedures [54].

\section{Trisodium Citrate Lock}

In the last decade, trisodium citrate has emerged as an alternative to heparin as a TC locking solution. Seven clinical trials reported citrate lock $(4 \%, 30 \%$, or $46.7 \%)$ to be equivalent or superior to heparin lock $(5,000-10,000 \mathrm{U} / \mathrm{mL})$ with respect to the thrombolytic therapy rates and number of TCs removed for flow problems (Table $1[6,9-12]$ ). In contrast, 2 studies reported unfavorable outcomes with citrate lock. The first was a small study (19 TCs) comparing citrate 5\% to heparin $(5000 \mathrm{U} / \mathrm{mL})$ [13]. The rate of thrombus aspirated from the TC was doubled with citrate (14\% versus $7 \%$, $P<0.001)$; however, the rate of thrombolytic therapy was not significantly different $(P=\mathrm{NS})$. In a larger $(n=232)$ recently published randomized controled trial comparing $46.7 \%$ citrate lock versus heparin lock $(5,000 \mathrm{U} / \mathrm{mL})$, the need for thrombolytic therapy was greater in the citrate group (8.2 versus 4.3/1000 days, $P<0.001$ ) [17]. One advantage of citrate lock is that the bleeding event rate is reported to be significantly lower. In the first study, which included both tunneled $(n=98)$ and nontunneled $(n=193)$ HD catheters in acute and chronic renal failure patients, citrate lock (30\%) was associated with a $70 \%$ reduction in major bleeding events when compared to heparin lock $(5,000 \mathrm{U} / \mathrm{mL}),(P=0.01)$ [15]. In the second study of 61 patients with HD TCs, there were significantly fewer systemic bleeding events using $4 \%$ citrate lock ( 7 in 32 patients) versus heparin lock $(5,000 \mathrm{U} / \mathrm{mL})(21$ in 29 patients $)(P=0.035)$ [12].

Citrate and other chelating agents have been shown, in vitro, to inhibit biofilm formation and growth of Staphylococcus aureus and Staphylococcus epidermidis at concentrations greater than $0.5 \%$. In contrast, heparin stimulated biofilm formation in this study [55]. Clinical trials using 4\% citrate lock have not shown to lower TC-related bacteremia (CRB) rates, with the exception of one study [10]. In a prospective, nonrandomized trial, a significant reduction in CRB rates was observed when the TC locking protocol changed from a time period using heparin lock to a period of citrate lock 4\% use; however, these findings are confounded by the concurrent initiation of a topical polyantibiotic ointment protocol, applied to the TC exit site, during the study period [10]. In two other studies comparing $4 \%$ citrate to heparin, there was no significant difference in CRB rates between locking agents $[11,12]$. The ability of citrate to inhibit biofilm formation and bacterial growth is highly concentration dependent. In an in vivo study, Ash et al. observed a reduction in CRB when concentrations of $23 \%$ citrate or higher were used as a HD TC locking solution [56]. In a large randomized controlled trial, 30\% citrate lock was associated with a significant reduction in the CRB rate $(1.1$ versus $4.1 / 1000$ TC-days, $P<0.001)$ and fewer admissions for TC-related infections ( 0.7 versus 2.7 per 1000 TC days) [15]. More recently, a randomized controlled trial by Power et al. using $46.7 \%$ citrate lock versus heparin lock $(5000 \mathrm{U} / \mathrm{mL})$, failed to show a difference in CRB rates; however, the CRB rate was lower than that reported in other series (0.7/1000 TC days), which may have resulted in underpowering this study [17].

An additional potential advantage of $4 \%$ citrate lock is the estimated cost savings calculated in 2 Canadian studies. An $80-85 \%$ reduction in costs was calculated using citrate in comparison to heparin $[10,11]$. In a 2008 position paper by the American Society of Diagnostic Interventional Nephrologists, the working group recommended that either 
heparin lock $1000 \mathrm{U} / \mathrm{mL}$ or $4 \%$ citrate lock be used in most TCs and that the injected volume not exceed the internal TC volume [57]. There are, however, advantages to citrate lock that make it a more desirable option, including lower bleeding risk, possible reduction in biofilm formation, avoidance of HAAb formation, lack of interference with prothrombin assays, and lower cost. At the present time, 4\% citrate is used in the majority of Canadian HD units where it is available as a prefilled syringe $(5 \mathrm{~mL})$. (Citralok, MEDXL, Montreal, QC, Canada) In the United States $4 \%$ citrate is currently available in larger volume bags $(250-500 \mathrm{mls})$ requiring preparation by the HD unit staff.

5.1. Novel Catheter Locking Solutions. Recently, Maki et al. reported the results of a multicentered, randomized, controlled trial using a novel catheter lock solution containing sodium citrate, methylene blue, methylparaben, and propylparaben (C-MB-P) in comparison to heparin catheter lock for HD TCs. The use of the C-MB-P solution was associated with a lower incidence of TC loss due to patency failure ( 0 versus $4, P=0.04$ ) and a lower rate of TC-related bacteremia (RR 0.29; CI 0.12-0.70; $P=0.005$ ) [58].

\section{Thrombolytic Agents as Catheter Locking Solutions}

Fibrinolytic agents have been studied as an alternative to heparin for use as a TC locking solution. The potential advantage of these agents is the prevention of TC-related infections and improved TC patency. In a meta-analysis of 5 randomized controlled trials in 991 cancer patients using TCs for chemotherapy, urokinaselock or flush was associated with a significant reduction in TC-related infection (HR 0.77, 95\% CI 0.60-0.98, $P=0.01$ ) [59]. In vitro, alteplase has been shown to modestly inhibit Staphylococcus aureus biofilm formation in concentrations $\geq 0.5 \mathrm{mg} / \mathrm{mL}$ [55]. Prevention of HD TC thrombosis using a tissue plasminogen activator (TPA) interdialytic lock was first evaluated in 2 small clinical studies. In comparison to alteplase lock $(1 \mathrm{mg} / \mathrm{mL})$, heparin lock $(5000 \mathrm{U} / \mathrm{mL})$ was associated with more frequent thromboses (O.R. 2.4, 95\% CI 1.5-4.0; $P=0.001$ ). Schenk et al., using a prospective randomized crossover design, evaluated the efficacy of TPA lock versus heparin lock $(1000 \mathrm{U} / \mathrm{mL})$ in 12 HD TCs [18]. TPA lock was associated with significantly improved blood flow rates, lower venous pressures, and fewer complications. In contrast, TC thrombosis, requiring fibrinolytic intervention, occurred in $20 \%$ of patients during the heparin period. There was no difference in bleeding or infectious events between the groups. The "Pre-CLOT" (Prevention of Catheter Lumen Occlusion with r-TPA versus heparin) randomly assigned 225 patients with a newly inserted TC to heparin (5000 U per milliliter) three times per week or recombinant tissue plasminogen activator (rt-PA) ( $1 \mathrm{mg}$ in each lumen) substituted for heparin at the midweek session (with heparin used in the other two sessions) [20]. TC malfunction occurred in 40 of the 115 patients assigned to heparin only $(34.8 \%)$ and 22 of the 110 patients assigned to rt-PA $(20.0 \%)$ (hazard ratio, $1.91 ; 95 \%$ confidence interval
(CI), 1.13 to $3.22 ; P=0.02)$. Catheter-related bacteremia occurred in 15 patients (13.0\%) assigned to heparin only, as compared with 5 (4.5\%) assigned to rt-PA (corresponding to 1.37 and 0.40 episodes per 1000 patient-days in the heparin and rt-PA groups, resp.; $P=0.02$ ). The risk of bacteremia from any cause was higher in the heparin group than in the rt-PA group by a factor of 3 (hazard ratio, 3.30; 95\% CI, 1.18 to $9.22 ; P=0.02$ ). The risk of adverse events, including bleeding, was similar in the two groups. The incremental cost of caring for patients with rt-PA as compared with heparin was $\$ 1,173$ per patient (Canadian dollars).

\section{Oral Agents for Prophylaxis to Inhibit Catheter Dysfunction}

The use of warfarin alone or in combination with an antiplatelet agent has been evaluated for primary prevention of HD TC dysfunction in 3 randomized controlled trials. The first study was a randomized placebo controlled trial which included $85 \mathrm{HD}$ patients receiving their first TC and compared fixed dose warfarin $(1 \mathrm{mg} /$ day $)$ to placebo [23]. This minidose of warfarin was previously shown to be associated with a $75 \%$ reduction in TC thrombosis rates in cancer patients [60]. Unfortunately, minidose warfarin was not associated with improvement in primary unassisted patency or assisted TC survival in HD TCs. There was no increase in bleeding events with mini-dose coumadin. Another important finding of this study was that an INR of $<1.00$ was associated with significantly greater risk of TC loss due to dysfunction (HR $=4.0,95 \%$ C.I. 1.1-14.5; $P=0.04$ ) and earlier need for thrombolytic therapy (H.R 2.8, 95\% CI 1.3-6.1, $P=0.009$ ) [17]. The second study compared low-intensity monitored warfarin (target INR 1.51.9) to placebo in HD patients with newly placed TCs [24]. Warfarin was ineffective in preventing TC dysfunction. The third study included 144 newly inserted HD TCs, compared low-intensity warfarin (targeted to an INR of $1.8-2.5)$ and ticlodipine $(250 \mathrm{mg} /$ day $)$ initiated within 12 hours after TC insertion (primary prevention) to a control group (ticlodipine alone) who received warfarin after the first thrombosis (secondary prevention) [27]. There was a significant reduction in TC thrombosis/dysfunction when the combined regimen of warfarin and ticlodipine was used as primary prevention compared to secondary prevention ( 0.16 versus 1.65 thrombotic events/patient year, $P<0.001$ ), improvement in TC flow rates, and fewer TC removals for dysfunction $(2.4 \%$ versus $17.5 \%, P<0.001)$. It should be noted, however, that more patients in the primary prevention group achieved adequate anticoagulation than in the secondary prevention group $(92 \%$ versus $65 \%, P<$ 0.05). There were no bleeding events associated with the warfarin/ticlodipine combination.

Warfarin use for secondary prevention was also evaluated by Zellweger et al. in a prospective study of $35 \mathrm{HD}$ patients considered high risk for TC dysfunction given low-intensity warfarin (INR 1.5-2.0) compared to low-risk TC patients [25]. Therapeutic warfarin with adequate anticoagulation was associated with improved dysfunction-free TC survival 
at 9 months $(47.1 \%)$ in comparison to that with inadequate anticoagulation $(8.1 \%)(P=0.01)$. In an observational study by Obialo et al., $63 \mathrm{HD}$ patients with TCs who were already receiving chronic aspirin $(n=21, \mathrm{~A}, 325 \mathrm{mg} /$ day $)$ or therapeutic warfarin $(n=11, \mathrm{~W}$, target INR 2-3) therapy for an underlying cardiovascular indication, and controls ( $n=31, \mathrm{C})$ not taking either medication were prospectively monitored [26]. Both aspirin and warfarin were associated with improved primary TC patency at 120 days in comparison controls (C) (A 91\%, W 73\%, C 29\% $(P<0.001))$. Gastrointestinal bleeding rates were significantly higher in those patients on aspirin and warfarin in comparison to controls (A 24\%, W 18\%, C 0\%, P<0.02), and elderly patients were at highest risk of bleeding (H.R. 1.14, 95\% C.I. 1.0-1.3, $P=0.008$ ).

In summary, the combination of low-intensity warfarin, to achieve a target INR of 1.5-2.0, with or without ticlodipine, has not been consistently shown to be efficacious in maintaining HD TC patency, particularly when used as primary prevention. Although this therapeutic combination was not associated with an increased bleeding risk in the trial by Colì et al., low-intensity warfarin was associated with a significant bleeding risk in HD patients when used for the prevention of TC [24] or arteriovenous graft thrombosis [61]. In this study, by Crowther et al., warfarin was associated with 6 major bleeding events (all patients were also on aspirin), whereas none in the placebo group had major bleeds $(P=0.03)$. Furthermore, warfarin had no effect on arteriovenous graft survival. The use of warfarin should be reserved for high-risk patients with recurrent TC dysfunction, using a low-intensity protocol (target INR of 1.5-2.0).

\section{Heparin-Coated Catheters}

Heparin-coated HD TCs (HCC) have not been shown to reduce the need for thrombolytic therapy or improve TC survival in 2 retrospective trials [21, 22]. However, in one of the studies, Jain et al. reported a significantly lower TCassociated bacteremia rate in the HCC group (34\% HCC versus $60 \%$ noncoated HD TCs, $P<0.001$ ) [22]. In the second study, Clark et al. found no difference in infection between HCC and noncoated HD TCs; however, the mean observation period was relatively brief; (48-74 days) [21].

\section{Conclusion}

TCs remain uniquely vital to provide not only shortterm renal replacement therapy but also long-term HD to thousands of patients every day. TC malfunction and infections are the two most important complications from TC and are responsible for higher morbidity and mortality in many dialysis patients. Recent studies have elucidated the importance of bacteria in the formation of biofilms and the development of fibrin sheaths that play a major role in the development of TC malfunction and TC-associated infections.
Most efforts to prevent dialysis TC malfunction have focused on the instillation of locking solutions in TCs. Heparin and citrate prevent clot formation in dialysis TCs and are the most commonly used TC lock solutions. Thrombolytic agents have been recently shown to be more effective in reducing TC-related infections and improving TC patency. Systemic administration of oral anticoagulation could be of benefit in selected patients at high risk for recurrent TC dysfunction, but is associated with greater bleeding risk. The role of antiplatelet agents for prevention of TC dysfunction has not been defined.

\section{Future Directions}

Important areas of future research include development of modified TCs with new designs and thrombosis-resistant properties and novel locking solutions and interventions to prevent or reduce biofilm and fibrin sheath formation. Furthermore, attaining a better understanding of the pathogenesis of biofilm and fibrin sheath development may allow for the development of novel therapies to manage these entities through translational research. Thus, other additional future areas of research could focus on (1) the molecular and genetic basis of biofilm and fibrin sheath development, (2) therapeutic agents that target the biofilm phenotype and community signaling-based agents that prevent the formation, or promote the detachment, of biofilms, and (3) whether a blood-based biomarker could be associated with the conversion from bacteria colonization into infection and fibrin sheath development [35, 47].

\section{References}

[1] National Kidney Foundation DOQI, "Clinical practice guidelines for vascular access," American Journal of Kidney Diseases, vol. 48, supplement 1, pp. S176-S247, 2006.

[2] US Renal Data System, USRDS 2010 Annual Data Report: Atlas of Chronic Kidney Disease and End-Stage Renal Disease in the United States, National Institutes of Health NIDDK, Bethesda, Md, USA, 2010.

[3] A. Besarab and R. Pandey, "Catheter management in HD patients: delivering adequate flow," Clinical Journal of the American Society of Nephrology, vol. 6, no. 1, pp. 227-234, 2011.

[4] A. Asif, D. Merrill, P. Briones, D. Roth, and G. A. Beathard, "Hemodialysis vascular access: percutaneous interventions by nephrologists," Seminars in Dialysis, vol. 17, no. 6, pp. 528534, 2004.

[5] E. Lacson, J. M. Lazarus, J. Himmelfarb, T. A. Ikizler, and R. M. Hakim, "Balancing fistula first with catheters last," American Journal of Kidney Diseases, vol. 50, no. 3, pp. 379-395, 2007.

[6] J. Davidson, P. Arnold, and F. Rivera, "Dual lumen catheters for dialysis," in Access for Dialysis, J. Davidson, Ed., Landes Bioscience, Georgetown, Ky, USA, 2002.

[7] C. M. Thomas, J. Zhang, T. H. Lim, N. Scott-Douglas, R. B. Hons, and B. R. Hemmelgam, "Alberta kidney disease network. Concentration of heparin-locking solution and risk of central venous HD catheter malfunction," ASAIO Journal, vol. 53, pp. 485-488, 2007.

[8] J. L. Holley and S. Bailey, "Catheter lock heparin concentration: effects on tissue plasminogen activator use in tunneled 
cuffed catheters," Hemodialysis International, vol. 11, no. 1, pp. 96-98, 2007.

[9] J. Buturovic, R. Ponikvar, A. Kandus, M. Boh, J. Klinkmann, and P. Ivanovitch, "Filling HD catheters in the interdialytic period: heparin versus citrate versus polygeline: a prospective randomized study," Artificial Organs, vol. 22, pp. 945-947, 1998.

[10] C. E. Lok, D. Appleton, C. Bhola, B. Khoo, and R. M. A. Richardson, "Trisodium citrate $4 \%$ - an alternative to heparin capping of haemodialysis catheters," Nephrology Dialysis Transplantation, vol. 22, no. 2, pp. 477-483, 2007.

[11] L. Grudzinski, P. Quinan, S. Kwok, and A. Pierratos, "Sodium citrate $4 \%$ locking solution for central venous dialysis catheters-an effective, more cost-efficient alternative to heparin," Nephrology Dialysis Transplantation, vol. 22, no. 2, pp. 471-476, 2007.

[12] J. M. MacRae, I. Dojcinovic, O. Djurdjev et al., "Citrate $4 \%$ versus heparin and the reduction of thrombosis study (CHARTS)," Clinical Journal of the American Society of Nephrology, vol. 3, no. 2, pp. 369-374, 2008.

[13] L. Hendrickx, D. Kuypers, P. Evenepoel, B. Maes, T. Messiaen, and Y. Vanrenterghem, "A comparative prospective study on the use of low concentrate citrate lock versus heparin lock in permanent dialysis catheters," International Journal of Artificial Organs, vol. 24, no. 4, pp. 208-211, 2001.

[14] K. J. F. Stas, J. Vanwalleghem, B. De Moor, and H. Keuleers, "Trisodium citrate $30 \%$ vs heparin $5 \%$ as catheter lock in the interdialytic period in twin- or double-lumen dialysis catheters for intermittent haemodialysis," Nephrology Dialysis Transplantation, vol. 16, no. 7, pp. 1521-1522, 2001.

[15] M. C. Wiejmer, M. A. van den Dorpel, P. J. G. Van de Ven et al., "Randomized, clinical trial comparison of trisodium citrate $30 \%$ and heparin as catheter-locking solution in HD patients," Journal of the American Society of Nephrology, vol. 16, pp. 2769-2777, 2005.

[16] B. Bayes, J. Bonal, and R. Romero, "Sodium citrate for filling haemodialysis catheters," Nephrology Dialysis Transplantation, vol. 14, no. 10, pp. 2532-2533, 1999.

[17] A. Power, N. Duncan, S. K. Singh et al., "Sodium citrate versus heparin catheter locks for cuffed central venous catheters: a single-center randomized controlled trial," American Journal of Kidney Diseases, vol. 53, no. 6, pp. 1034-1041, 2009.

[18] P. Schenk, A. R. Rosenkranz, G. Wölfl, W. H. Hörl, and O. Traindl, "Recombinant tissue plasminogen activator is a useful alternative to heparin in priming Quinton Permcath," American Journal of Kidney Diseases, vol. 35, no. 1, pp. 130136, 2000.

[19] N. S. Gittins, Y. L. Hunter-Blair, J. N. S. Matthews, and M. G. Coulthard, "Comparison of alteplase and heparin in maintaining the patency of paediatric central venous haemodialysis lines: a randomised controlled trial," Archives of Disease in Childhood, vol. 92, no. 6, pp. 499-501, 2007.

[20] B. R. Hemmelgarn, L. M. Moist, C. E. Lok et al., "Prevention of dialysis catheter malfunction with recombinant tissue plasminogen activator," The New England Journal of Medicine, vol. 364, pp. 303-312, 2011.

[21] T. W. I Clark, D. Jacobs, H. W. Charles et al., "Comparison of heparin-coated and conventional split-tip HD catheters," CardioVascular and Interventional Radiology, vol. 32, pp. 703706, 2009.

[22] G. Jain, M. Allon, S. Saddekni, J. Barker-Finkel, and I. D. Maya, "Does heparin coating improve patency or reduce infection of tunneled dialysis catheters?" Clinical Journal of the American Society of Nephrology, vol. 4, no. 11, pp. 1787-1790, 2009.
[23] M. H. Mokrzycki, K. Jean-Jerome, H. Rush, M. P. Zdunek, and S. O. Rosenberg, "A randomized trial of minidose warfarin for the prevention of late malfunction in tunneled, cuffed hemodialysis catheters," Kidney International, vol. 59, no. 5, pp. 1935-1942, 2001.

[24] T. J. Wilkieson, A. J. Ingram, M. A. Crowther et al., "Lowintensity adjusted-dose warfarin for the prevention of HD catheter failure: a randomized controlled trial," Clinical Journal of the American Society of Nephrology, vol. 6, pp. 10181024, 2011.

[25] M. Zellweger, J. Bouchard, S. Raymond-Carrier, A. LaforestRenald, S. Quérin, and F. Madore, "Systemic anticoagulation and prevention of hemodialysis catheter malfunction," ASAIO Journal, vol. 51, no. 4, pp. 360-365, 2005.

[26] C. I. Obialo, A. C. Conner, and L. F. Lebon, "Maintaining patency of tunneled hemodialysis catheters-efficacy of aspirin compared to warfarin," Scandinavian Journal of Urology and Nephrology, vol. 37, no. 2, pp. 172-176, 2003.

[27] L. Colì, G. Donati, G. Cianciolo et al., "Anticoagulation therapy for the prevention of hemodialysis tunneled cuffed catheter (TCC) thrombosis," Journal of Vascular Access, vol. 7, no. 3, pp. 118-122, 2006.

[28] J. Work, "Chronic catheter placement," Seminars in Dialysis, vol. 14, no. 6, pp. 436-440, 2001.

[29] P. A. Conz, D. Dissegna, M. P. Rodighiero, and G. La Greca, "Cannulation of the internal jugular vein: comparison of the classic Seldinger technique and an ultrasound guided method," Journal of Nephrology, vol. 10, no. 6, pp. 311-313, 1997.

[30] R. Vanholder and S. Ringoir, "Vascular access for HD," American Journal of Kidney Diseases, vol. 25, pp. 732-737, 1995.

[31] J. S. Lameris, P. J. M. Post, H. M. Zonderland, P. G. Gerritsen, M. C. Kappers-Klunne, and H. E. Schutte, "Percutaneous placement of Hickman catheters: comparison of sonographically guided and blind techniques," American Journal of Roentgenology, vol. 155, no. 5, pp. 1097-1099, 1990.

[32] A. R. Forauer and J. F. Glockner, "Importance of US findings in access planning during jugular vein hemodialysis catheter placements," Journal of Vascular and Interventional Radiology, vol. 11, no. 2, part 1, pp. 233-238, 2000.

[33] S. J. Schwab, L. D. Quarles, J. P. Middleton, R. H. Cohan, M. Saeed, and V. W. Dennis, "Hemodialysis-associated subclavian vein stenosis," Kidney International, vol. 33, no. 6, pp. 11561159, 1988.

[34] R. M. Donlan and J. W. Costerton, "Biofilms: survival mechanisms of clinically relevant microorganisms," Clinical Microbiology Reviews, vol. 15, no. 2, pp. 167-193, 2002.

[35] J. W. Costerton, P. S. Stewart, and E. P. Greenberg, "Bacterial biofilms: a common cause of persistent infections," Science, vol. 284, no. 5418, pp. 1318-1322, 1999.

[36] J. W. Costerton, K. J. Cheng, G. G. Geesey et al., "Bacterial biofilms in nature and disease," Annual Review of Microbiology, vol. 41, pp. 435-464, 1987.

[37] M. K. Dasgupta, "Biofilms and infection in dialysis patients," Seminars in Dialysis, vol. 15, no. 5, pp. 338-346, 2002.

[38] L. Passerini, K. Lam, J. W. Costerton, and E. G. King, "Biofilms on indwelling vascular catheters," Critical Care Medicine, vol. 20, no. 5, pp. 665-673, 1992.

[39] E. Anaissie, G. Samonis, D. Kontoyiannis et al., "Role of catheter colonization and infrequent hematogenous seeding in catheter-related infections," European Journal of Clinical Microbiology and Infectious Diseases, vol. 14, no. 2, pp. 134-137, 1995.

[40] I. Raad, W. Costerton, U. Sabharwal, M. Sacilowski, E. Anaissie, and G. P. Bodey, "Ultrastructural analysis of indwelling 
vascular catheters: a quantitative relationship between luminal colonization and duration of placement," Journal of Infectious Diseases, vol. 168, no. 2, pp. 400-407, 1993.

[41] A. Dwyer, "Surface-treated catheters-a review," Seminars in Dialysis, vol. 21, no. 6, pp. 542-546, 2008.

[42] S. Faintuch and G. M. M. Salazar, "Malfunction of dialysis catheters: management of fibrin sheath and related problems," Techniques in Vascular and Interventional Radiology, vol. 11, no. 3, pp. 195-200, 2008.

[43] J. R. Mehall, D. A. Saltzman, R. J. Jackson, and S. D. Smith, "Fibrin sheath enhances central venous catheter infection," Critical Care Medicine, vol. 30, no. 4, pp. 908-912, 2002.

[44] O. Liangos, A. Gul, N. E. Madias, and B. L. Jaber, "Long-term management of the tunneled venous catheter," Seminars in Dialysis, vol. 19, no. 2, pp. 158-164, 2006.

[45] H. Ishida, Y. Ishida, Y. Kurosaka, T. Otani, K. Sato, and H. Kobayashi, "In vitro and in vivo activities of levofloxacin against biofilm- producing Pseudomonas aeruginosa," Antimicrobial Agents and Chemotherapy, vol. 42, no. 7, pp. 1641-1645, 1998.

[46] J. W. Bosma, C. E. Siegert, P. G. Peerbooms, and M. C. Weijmer, "Reduction of biofilm formation with trisodium citrate in haemodialysis catheters: a randomized controlled trial," Nephrology, Dialysis, Transplantation, vol. 25, pp. 1213 1217, 2010.

[47] S. M. Jones, P. Ravani, B. R. Hemmelgarn, D. Muruve, and J. M. Macrae, "Morphometric and biological characterization of biofilm in tunneled HD catheters," American Journal of Kidney Diseases, vol. 57, pp. 449-455, 2011.

[48] A. S. Yevzlin, R. J. Sanchez, J. G. Hiatt et al., "Concentrated heparin lock is associated with major bleeding complications after tunneled hemodialysis catheter placement," Seminars in Dialysis, vol. 20, no. 4, pp. 351-354, 2007.

[49] M. Sungur, E. Eryuksel, S. Yavas, A. Bihorac, A. J. Layon, and L. Caruso, "Exit of catheter lock solutions from double lumen acute haemodialysis catheters_-an in vitro study," Nephrology Dialysis Transplantation, vol. 22, no. 12, pp. 3533-3537, 2007.

[50] M. Agharazii, I. Plamondon, M. Lebel, P. Douville, and S. Desmeules, "Estimation of heparin leak into the systemic circulation after central venous catheter heparin lock," Nephrology Dialysis Transplantation, vol. 20, pp. 1238-1240, 2005.

[51] I. Markota, D. Markota, and M. Tomic, "Measuring of the heparin leakage into the circulation from central venous catheters-an in vivo study," Nephrology Dialysis Transplantation, vol. 24, no. 5, pp. 1550-1553, 2009.

[52] H. D. Polaschegg and C. Shah, "Overspill of catheter locking solution: safety and efficacy aspects," ASAIO Journal, vol. 49, no. 6, pp. 713-715, 2003.

[53] H. Karaaslan, P. Peyronnet, D. Benevent, C. Lagarde, M. Rince, and C. Leroux-Robert, "Risk of heparin lock-related bleeding when using indwelling venous catheter in haemodialysis," Nephrology Dialysis Transplantation, vol. 16, no. 10, pp. 2072 2074, 2001.

[54] N. T. Oran and I. Eser, "Impact of heparin locking frequency on preventing temporary dialysis catheter dysfunction in haemodialysis patients," Journal of Renal Care, vol. 34, no. 4, pp. 199-202, 2008.

[55] R. M. Q. Shanks, J. L. Sargent, R. M. Martinez, M. L. Graber, and G. A. O'Toole, "Catheter lock solutions influence staphylococcal biofilm formation on abiotic surfaces," Nephrology Dialysis Transplantation, vol. 21, no. 8, pp. 2247-2255, 2006.
[56] S. R. Ash, R. A. Mankus, J. M. Sutton et al., "Concentrated sodium citrate (23\%) for catheter lock," Hemodialysis International, vol. 4, pp. 22-31, 2000.

[57] J. E. Moran and S. R. Ash, "Locking solutions for HD catheters: heparin and citrate-a position paper by ASDIN," Seminars in Dialysis, vol. 21, pp. 490-492, 2008.

[58] D. G. Maki, S. R. Ash, R. K. Winger, and P. Lavin, "A novel antimicrobial and antithrombotic lock solution for hemodialysis catheters: a multi-center, controlled, randomized trial," Critical Care Medicine, vol. 39, pp. 613-620, 2011.

[59] S. Kethireddy and N. Safdar, "Urokinase lock or flush solution for prevention of bloodstream infections associated with central venous catheters for chemotherapy: a meta-analysis of prospective randomized trials," Journal of Vascular Access, vol. 9, no. 1, pp. 51-57, 2008.

[60] M. M. Bern, J. J. Lokich, S. R. Wallach et al., "Very low doses of warfarin can prevent thrombosis in central venous catheters. A randomized prospective trial," Annals of Internal Medicine, vol. 112, no. 6, pp. 423-428, 1990.

[61] M. A. Crowther, C. M. Clase, P. J. Margetts et al., "Lowintensity warfarin is ineffective for the prevention of PTFE graft failure in patients on hemodialysis: a randomized controlled trial," Journal of the American Society of Nephrology, vol. 13, no. 9, pp. 2331-2337, 2002. 


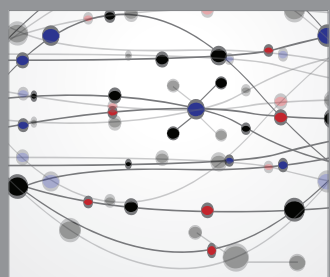

The Scientific World Journal
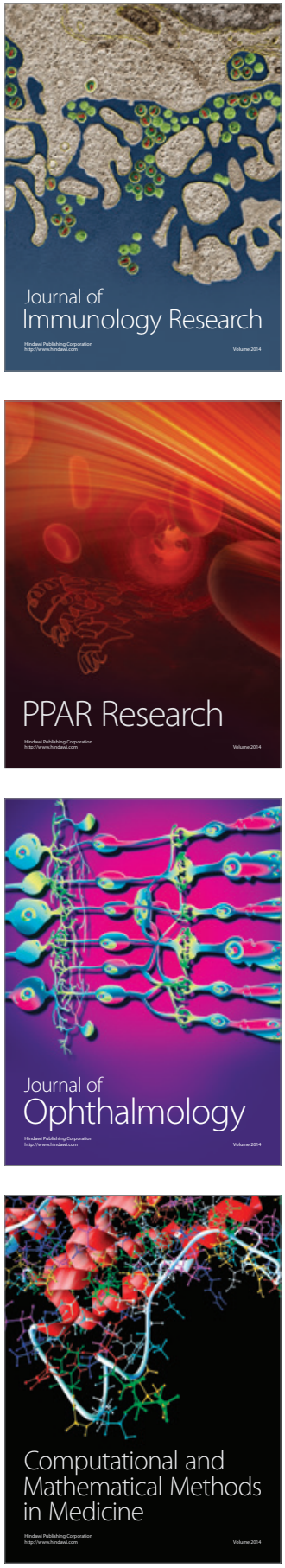

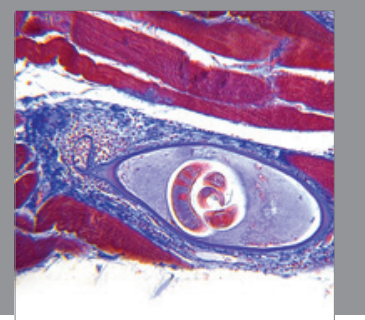

Gastroenterology

Research and Practice
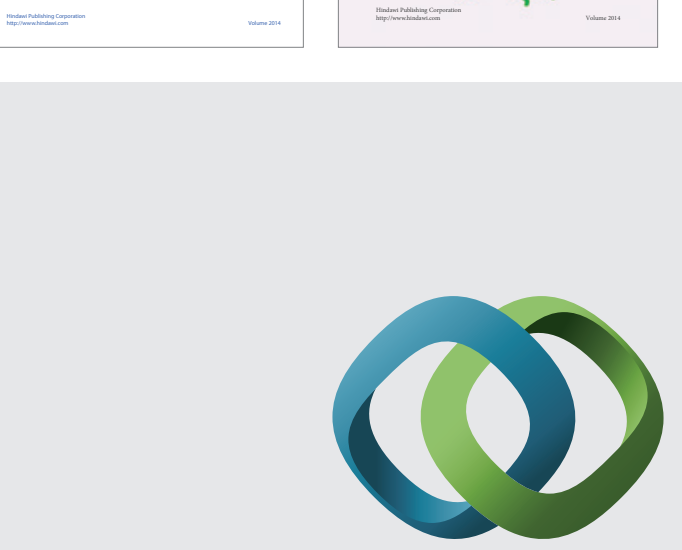

\section{Hindawi}

Submit your manuscripts at

http://www.hindawi.com
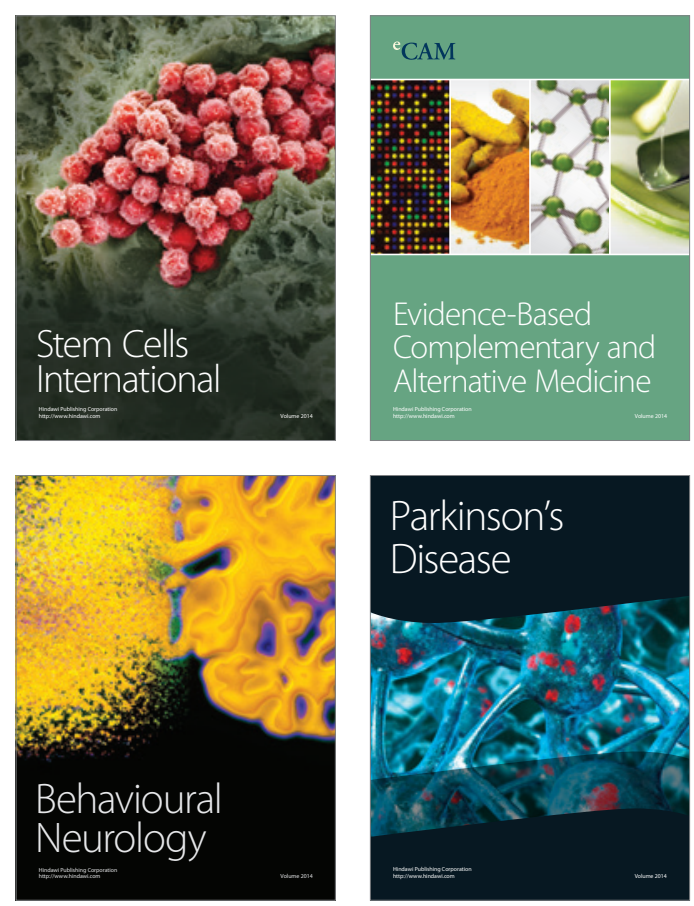

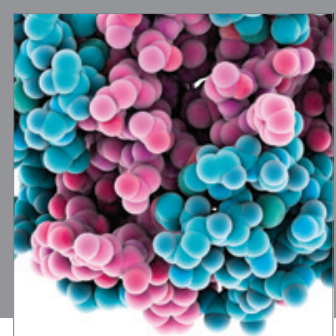

Journal of
Diabetes Research

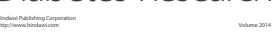

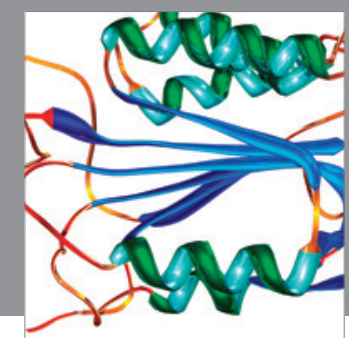

Disease Markers
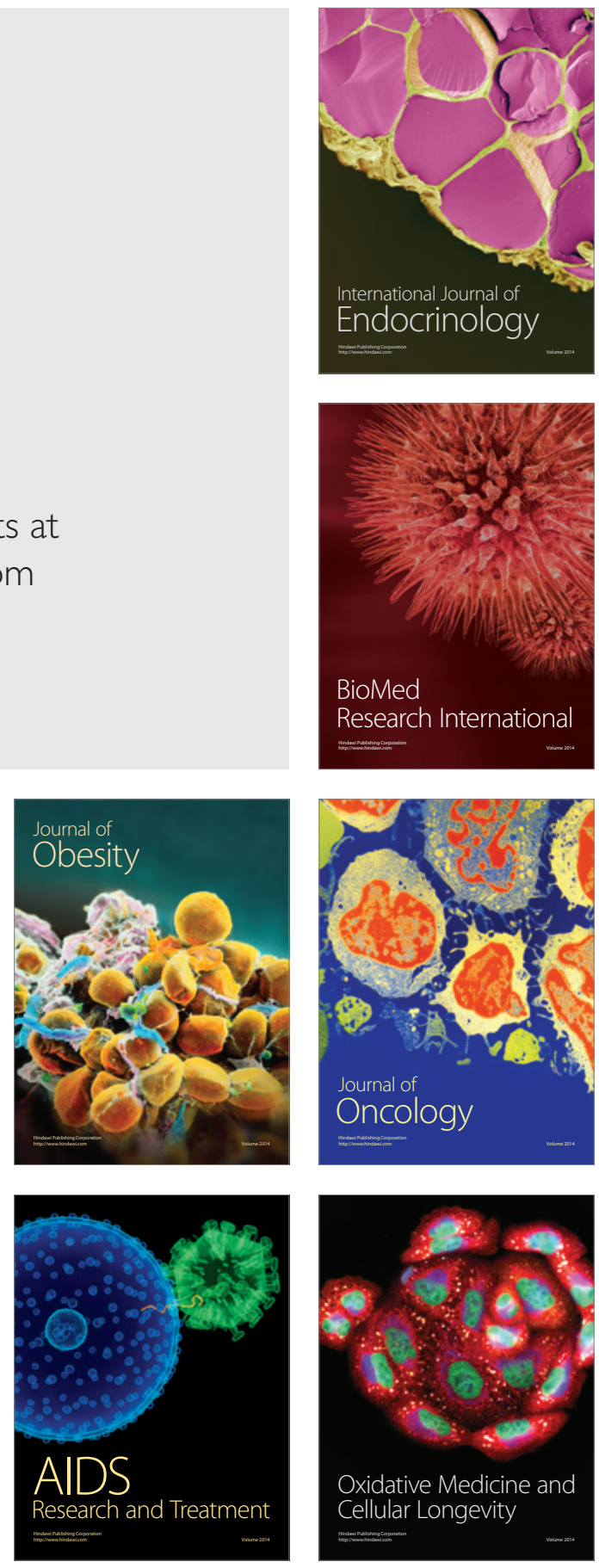